\title{
TOWARDS MINIMAL BINDING VARIETIES OF LATTICES
}

\author{
VÁCLAV KOUBEK
}

Introduction. By classical results of Ambrust and Schmidt, every monoid is isomorphic to the endomorphism monoid of an algebra. Thus if we want to investigate in which varieties (or generally, in a concrete category) each monoid is isomorphic to the endomorphism monoid of an algebra in this variety (or an object of this category) then the important role is played by the concept of a binding category (see the definition below). Moreover, as shows the Hedrlín-Kucera Theorem [13] if the set-theoretical assumption (M) holds (i.e., there is an infinite cardinal $\alpha$ such that each ultrafilter closed under $\alpha$ intersections is trivial) every concrete category can be fully embedded into any binding category. Other properties of binding categories are in $[7,11,13]$. A list of the most important binding categories and the properties of binding categories can be found in an excellent monograph [13].

This paper is devoted to binding varieties of bounded lattices and $(0,1)$-homomorphisms. Grätzer and Sichler [5] proved that the variety of all bounded lattices is binding. Adams and Sichler [1] strengthened this result by proving that there is a locally finite variety $\mathscr{A}$ of bounded lattices (it will be defined later) that is binding and asked whether $\mathscr{A}$ is the smallest binding variety of bounded lattices. The aim of this paper is a negative answer to this question. We define two varieties $\mathscr{A}(B)^{*}$ and $\mathscr{A}(B)_{*}$ of bounded lattices and prove that they are binding and their intersection is the variety generated by $N_{5}$; in this variety each lattice has a prime ideal and thus it is not binding, see [13]. Therefore, there is no smallest binding variety of bounded lattices. The following problem is open: Are the varieties $\mathscr{A}(B)_{*}$ and $\mathscr{A}(B)^{*}$ minimal binding varieties of bounded lattices?

The proof of the main theorem is not based on the existence of complement pairs, in contrast to [1] and [5]. In fact, our lattices have exactly one complement pair, 0 and 1 .

The paper has four parts. The first part consists of standard definitions and facts in Graph Theory, Category Theory and Lattice Theory. In the second part auxiliary graph constructions are given; a special binding full subcategory of the category of all graphs is constructed. The third part

Received July 13, 1982, and in revised form April 22, 1983. The support of the National Research Council of Canada is gratefully acknowledged. 
contains a proof of the main theorem: $\mathscr{A}(B) *$ and $\mathscr{A}(B)^{*}$ are binding. The last part continues [2] by a description of the lattices of subvarieties of $\mathscr{A}(B)_{*}$ or $\mathscr{A}(B)^{*}$. In particular, these lattices are countable in contrast to the lattice of subvarieties of $\mathscr{A}$ which is uncountable.

1. Preliminaries. For a set $X$, denote by $|X|$ its cardinality. Each ordinal $\alpha$ is identified with the set of all ordinals less than $\alpha$. Cardinals are identified with initial ordinals. The set of all natural numbers (i.e., the set of all finite cardinals) is denoted by $\mathbf{N}$.

Since the paper uses notions from four different fields, we recall some basic notations used in these fields.

Graph theory. A graph is a pair $(X, R)$ where $X$ is a set and $R$ is a set of some two-point subsets of $X$. Elements of $X$ are called vertices, elements of $R$ are called edges. If $(X, R),(Y, S)$ are graphs then a mapping $f: X \rightarrow Y$ is a compatible mapping from $(X, R)$ to $(Y, S)$ if $\{f(x), f(y)\} \in S$ for each $\{x, y\} \in R$. The category of all graphs and compatible mappings is denoted by Gra. A directed loop free graph is a pair $(X, R)$ where $X$ is a set and

$$
R \subset X \times X-\{(x, x) ; x \in X\} .
$$

If $(X, R),(Y, S)$ are directed loop free graphs then a mapping $f: X \rightarrow Y$ is a compatible mapping from $(X, R)$ to $(Y, S)$ if

$$
(f(x), f(y)) \in S \text { for each }(x, y) \in R .
$$

The category of all directed loop free graphs and compatible mappings is denoted by DiGr. We recall that a subset $T$ of a graph $(X, R)$ is called a component of $(X, R)$ if $T$ is a maximal set (with respect to inclusion) such that for each pair $x, y \in T$ there is a sequence $x=x_{0}, x_{1}, \ldots, x_{n}=y$ with

$$
\left\{x_{i}, x_{i+1}\right\} \in R \text { for each } i=0,1, \ldots, n-1 .
$$

The set of all components forms a decomposition of $X$. A subset $T$ of a graph $(X, R)$ is independent if for each pair of vertices $u, v$ of $T,\{u, v\} \notin R$. For a vertex $x$ of $(X, R)$ the degree of $x$ is the cardinality of the set $\{y ;\{x$, $y\} \in R\}$. If $T$ is a subset of $(X, R)$ then $(T,\{\{x, y\} \in R ; x, y \in T\})$ is a full subgraph of $(X, R)$ induced on $T$ (or shortly, on a set $T$ ). A graph $(X, R)$ is called complete if $R$ is the set of all two-point subsets of $X$. In particular, a three-point subset $T$ of $X$ is called a triangle in $(X, R)$ if the full subgraph induced on $T$ is complete. A graph $(X, R)$ is called a complete bipartite graph, if there is a decomposition $X_{1}, X_{2}$ of $X$ such that

$$
R=\left\{\{x, y\} ; x \in X_{1}, y \in X_{2}\right\} .
$$


Category theory. A functor $F: \mathscr{L} \rightarrow \mathscr{K}$ is called a full embedding if for each pair $a, b$ of $\mathscr{L}$-objects it holds that:

(i) if $f, g: a \rightarrow b$ are distinct $\mathscr{L}$-morphisms then $F f \neq F g$ (i.e., $F$ is faithful);

(ii) if $f: F a \rightarrow F b$ is a $\mathscr{K}$-morphism then $f=F g$ for an $\mathscr{L}$-morphism $g: a \rightarrow b$ (i.e., $F$ is full).

A pair $(\mathscr{L}, F)$ is a concrete category where $\mathscr{L}$ is a category, $F$ is a faithful functor from $\mathscr{L}$ to a category of all sets and mappings (usually, if $F$ is clear we omit it).

An object $A$ of a category $\mathscr{K}$ is called rigid if it has exactly one endomorphism, the identity of $A$.

A concrete category is called a binding (or universal) category if every category of algebras and their homomorphisms can be fully embedded into it. This is well-known (see e.g. [13]).

\section{THEOREM 1.1. Gra and DiGr are binding.}

Lattice theory. In this paper "lattice" means a bounded lattice (i.e., a lattice with the least element 0 and the greatest element 1), "homomorphism" means a $(0,1)$-homomorphism; i.e., a homomorphism preserving 0 and 1; "sublattice" means a $(0,1)$-sublattice; i.e., a sublattice with the same 0 and 1 as the original lattice. A complemented pair of a lattice $L$ is a pair $a, b$ of elements of $L$ with $a \cdot b=0, a+b=1$ (we shall denote the meet as $\cdot$, the join as + ). If $L$ is a lattice, then an element $a$ of $L$ is an atom if $a \neq 0$ and for every element $b \in L, a \geqq b>0$ implies $a=b$. A subset $X$ of a lattice $L$ is called an order-ideal if $y \in X$ whenever there is $x$ $\in X$ with $x \geqq y$. For a lattice $L$ and an element $a \in L$, the set $\{y \in L ; y$ $\leqq a\}$ is called the principal ideal generated by $a$. An element $a$ of a lattice $L$ is called join-irreducible (or meet-irreducible) if $a=x+y$ (or $a=x \cdot y$ ) in $L$ implies $a=x$ or $a=y$. For a graph $\mathscr{G}=(X, R)$, denote by $I(\mathscr{G})_{*}$ the set of all finite independent sets of $(X, R)$ ordered by the inclusion with an added new element 1 . Then $I(\mathscr{G})_{*}$ is a lattice. In particular, if $\mathscr{G}$ is a complete bipartite graph with a decomposition on $X_{1}, X_{2}$ of $X$ such that

$$
R=\left\{\{x, y\} ; x \in X_{1}, y \in X_{2}\right\}
$$

then $I(\mathscr{G})_{*}$ is the set of all finite subsets of $X_{1}$ or of $X_{2}$ ordered by the inclusion plus a greatest element added. The dual lattice of $I(\mathscr{G})_{*}$ is denoted by $I(\mathscr{G})^{*}$. Then $\mathscr{A}$ is the smallest variety containing $I(\mathscr{G})_{*}$ and $I(\mathscr{G})^{*}$ for each graph $\mathscr{G}$. Define $\mathscr{A}(B)_{*}\left(\right.$ or $\left.\mathscr{A}(B)^{*}\right)$ the smallest variety containing all $I(\mathscr{G})_{*}\left(\right.$ or $I(\mathscr{G})^{*}$, resp.) for each complete bipartite graph.

Universal algebra. A variety $\mathscr{V}$ is locally finite if each finitely generated algebra in $\mathscr{V}$ is finite. An algebra $A$ is called subdirectly irreducible if it has a least non-identical congruence. 
2. A graph-theoretical representation. The aim of this section is to show that a subcategory of Gra with certain properties is binding. This result will be used in the next section for a construction of full embeddings of a suitable full subcategory of Gra into varieties $\mathscr{A}(B) *$ and $\mathscr{A}(B)^{*}$ of lattices. The theorem is a modification of the results and proofs in [10]. First we give a construction of a rigid graph with suitable properties. This graph will be used for the proof of Theorem 2.6 which describes a required subcategory $\mathbf{C}$ of Gra.

Construction 2.1. By induction, we shall construct a sequence of graphs $\left(Z_{i}, S_{i}\right)_{i \in \mathrm{N}}$ such that if $i<j$ then $\left(Z_{i}, S_{i}\right)$ is a full subgraph of $\left(Z_{j}, S_{j}\right)$. First choose an increasing one-to-one sequence $\left\{\alpha_{i}\right\}_{i \in \mathrm{N}}$ of infinite cardinals. Let $\left(Z_{0}, S_{0}\right)$ be the complete graph on the set $Z_{0}=\alpha_{0}$. To construct $\left(Z_{1}, S_{1}\right)$, first choose a rigid graph $\left(Z_{0}, P_{0}\right)$. For every edge $r=$ $\{x, y\} \in P_{0}$ we add a copy of the complete graph $\left(\alpha_{1}, C_{1}\right)$ and connect each point $a \in \alpha_{1}$ by edges to $x$ and $y$. Precisely, put

$$
\begin{aligned}
& Z_{1}=Z_{0} \cup\left(\alpha_{1} \times P_{0}\right), \\
& S_{1}=S_{0} \cup\left\{\{(a, r),(b, r)\} ; a, b \in \alpha_{1}, r \in P_{0}\right\} \\
& \cup\left\{\{(a, r), x\} ; a \in \alpha_{1}, r \in P_{0}, x \in r\right\} .
\end{aligned}
$$

Now we proceed by induction. If $\left(Z_{i}, S_{i}\right), i>0$ is constructed, we denote by $\Omega_{i}$ the set of all components of the full subgraph of $\left(Z_{i}, S_{i}\right)$ on the set $Z_{i}$ $-Z_{i-1}$. For each $W \in \Omega_{i}$ we choose a rigid graph $\left(W, P_{i, W}\right)$ and we form $\left(Z_{i+1}, S_{i+1}\right)$ such that for each edge $r=\{x, y\} \in P_{i, W}$ we add a copy of the complete graph $\left(\alpha_{i+1}, C_{i+1}\right)$ and each point $a$ of $\alpha_{i+1}$ we connect to $x$ and $y$ by edges. Precisely, put

$$
\begin{aligned}
& Z_{i+1}=Z_{i} \cup\left(\alpha_{i+1} \times \cup\left\{P_{i, W} ; W \in \Omega_{i}\right\}\right), \\
& S_{i+1}=S_{i} \cup\{\{(a, r),(b, r)\} ; \\
& \left.a, b \in \alpha_{i+1}, r \in P_{i, W} \text { for } W \in \Omega_{i}\right\} \\
& \cup\{\{(a, r), x\} ; \\
& \left.a \in \alpha_{i+1}, r \in P_{i, W} \text { for } W \in \Omega_{i}, x \in r\right\} .
\end{aligned}
$$

Put

$$
Z=\cup\left\{Z_{i} ; i \in \mathbf{N}\right\}, \quad \bar{S}=\cup\left\{S_{i} ; i \in \mathbf{N}\right\} .
$$

To form the graph $(Z, S)$ we add new edges to $\bar{S}$. For this purpose we choose a one-to-one mapping

$$
\varphi_{n}: Z_{0} \rightarrow \cup\left\{P_{n, W} ; W \in \Omega_{n}\right\} \text { for every } n \geqq 3
$$

(it is possible because $\left|P_{n, W}\right| \geqq \alpha_{n}$ for every $n$ ) and put

$$
S=\bar{S} \cup\left\{\left\{x,\left(a, \varphi_{n}(x)\right)\right\} ; a \in \alpha_{n+1}, x \in Z_{0}, n \in \mathbf{N}, n \geqq 3\right\} .
$$


Proposition 2.2. The graph $(Z, S)$ satisfies the following:

a) for each edge $\{x, y\} \in S$ there are infinitely many vertices $z \in Z$ such that $\{x, y, z\}$ is a triangle in $(Z, S)$;

b) assume that for every $n \in \mathbf{N}$ and every $W \in \Omega_{n}$ every vertex of the graph $\left(W, P_{n, W}\right)$ has an infinite degree then for every $z \in Z$ there is an infinite subset $T_{z}$ of $Z$ such that:

b1) if $t \in T_{z}$ then $\{t, z\} \in S$;

b2) for every $y \in Z$ and for every pair $t, u \in T_{z}, t \neq u$ either

$$
y=z \text { or }\{y, t\} \notin S \text { or }\{y, u\} \notin S
$$

c) there is an infinite set $T$ of disjoint triangles in $(Z, S)$, i.e., if $\{x, y, z\}$ and $\{t, u, v\}$ are distinct triangles in $T$ then

$$
\{x, y, z\} \cap\{t, u, v\}=\emptyset
$$

d) each vertex of $(Z, S)$ has an infinite degree in $(Z, S)$;

e) for each pair $x, y$ of vertices of $Z$ there is a finite sequence $T_{0}$, $T_{1}, \ldots, T_{n}$ of subsets of $Z$ with

$$
x \in T_{0}, y \in T_{n},\left|T_{i} \cap T_{i+1}\right| \geqq 2
$$

for each $i=0,1, \ldots, n-1$ such that the full subgraph of $(Z, S)$ induced on $T_{i}$ for each $i=0,1, \ldots, n$ is complete;

f) $(Z, S)$ is a rigid graph;

g) for each edge $\{x, y\} \in S_{i}-S_{-1}$ for $i \geqq 0$ (we put $S_{-1}=0$ ) there is a subset $T$ of $Z$ with $x, y \in T,|T|=\alpha_{i}$ such that the full subgraph of $(Z, S)$ induced on $T$ is complete;

h) for each subset $T$ of $Z$ such that the full subgraph of $(Z, S)$ induced on $T$ is complete, if $T \cap Z_{i} \neq \emptyset$ for an $i>0$ then $|T| \leqq \alpha_{i+1}$;

i) for each vertex $x \in Z_{0}$ and for each $i \in \mathbf{N}$ there is a subset $T \subset Z$ with $x \in T,|T|=\alpha_{i}$ such that the full subgraph of $(Z, S)$ induced on $T$ is complete;

j) $x \in Z_{n}-Z_{n-1}$ for $n>0$ if and only if a shortest sequence $T_{0}$, $T_{1}, \ldots, T_{k}$ of subsets of $Z$ with $x \in T_{0},\left|T_{k} \cap Z_{0}\right|>1$, and $\left|T_{i} \cap T_{i+1}\right|>$ 1 for every $i=0,1, \ldots, k-1$ such that the full subgraph of $(Z, S)$ induced on $T_{i}$ is complete for every $i$ has length $n$.

Proof. The verification of a), b), c), d), e), g), h), i) and j) is a straightforward calculation based on the construction of $(Z, S)$. We verify f). If $f:(Z, S) \rightarrow(Z, S)$ is a compatible mapping we get by h) and i) that $f\left(Z_{0}\right) \subset Z_{0}$ because $f$ preserves complete graphs. By j) we get that $f\left(Z_{i}\right) \subset$ $Z_{i}$ for each $i$ and by g) and h) we obtain that

$$
f\left(Z_{i}-Z_{i-1}\right) \subset Z_{i}-Z_{i-1} \text { for every } i \in \mathbf{N} .
$$

We prove by induction over $i$ that $f$ is the identity. For a pair $x, y \in Z_{0}$ we have $\{x, y\} \in P_{0}$ if and only if there is a subset $T$ of $Z$ with $x, y \in T,|T|$ $=\alpha_{1}$ such that the full subgraph of $(Z, S)$ induced on $T$ is complete. Thus 
the restriction of $f$ to $Z_{0}$ is an endomorphism of $\left(Z_{0}, P_{0}\right)$; hence $f / Z_{0}$ is the identity. Assume that $f / Z_{i}$ is the identity for some $i \in \mathbf{N}$. Let $\Omega_{i+1}$ be the set of all components of the full subgraph of $(Z, S)$ induced on $Z_{i+1}-Z_{i}$. According to the definition of $\left(Z_{i+1}, S_{i+1}\right)$ for each $W \in \Omega_{i+1}$ there is a pair $x_{W}, y_{W} \in Z_{i}$ such that for each $w \in W$ and for each $z \in Z_{i}$ we have $\{w, z\} \in S$ if and only if $z \in\left\{x_{W}, y_{W}\right\}$. Since $f\left(x_{W}\right)=x_{W}$ and $f\left(y_{W}\right)=$ $y_{W}$ we get $f(W) \subset W$ for each $W \in \Omega_{i+1}$. For a pair $x, y \in W \in \Omega_{i+1}$ we have that $\{x, y\} \in P_{i+1, W}$ if and only if there is a subset $T$ of $Z$ with $x, y$ $\in T,|T|=\alpha_{i+2}$ such that the full subgraph of $(Z, S)$ induced on $T$ is complete. Thus the restriction of $f$ to $W$ is an endomorphism of ( $W$, $\left.P_{i+1, W}\right)$. Hence $f / W$ is the identity. Since $W$ is an arbitrary component of the full subgraph of $(Z, S)$ induced on $Z_{i+1}-Z_{i}$, by the induction hypothesis we conclude that $f / Z_{i+1}$ is the identity. Thus $(Z, S)$ is rigid.

If we combine b) and d) of Proposition 2.2 we get:

COROLlary 2.3. There is a rigid graph $(Z, S)$ such that:

a) for every edge $\{x, y\} \in S$ there are infinitely many vertices $z \in Z$ such that $\{x, y, z\}$ is a triangle in $(Z, S)$;

b) for every vertex $z \in Z$ there is an infinite subset $T_{z}$ of $Z$ such that for each $t \in T_{z}$ we have $\{t, z\} \in S$ and for each vertex $y \in Z-\{z\}$ and for each pair $u, t \in T_{z}, u \neq t$ either $\{y, t\} \notin S$ or $\{y, u\} \notin S$;

c) there is an infinite set $T$ of disjoint triangles in $(Z, S)$;

d) for each pair $\{x, y\}$ of vertices in $Z$ there is a finite sequence of subsets $T_{0}, T_{1}, \ldots, T_{n}$ of $Z$ with $x \in T_{0}, y \in T_{n},\left|T_{i} \cap T_{i+1}\right|>1$ for each $i=0$, $1, \ldots, n-1$ such that the full subgraph of $(Z, S)$ induced on $T_{i}$ is complete for each $i=0,1, \ldots, n$.

We use a standard šíp construction, see e.g. [9], [12], [13]. A quadruple $(X, R, a, b)$ is called a šp if $(X, R)$ is a graph and $a, b$ are vertices of $(X$, $R)$.

Construction 2.4. Let $(X, R, a, b)$ be a šíp, then we define a functor $(X, R$, $a, b)^{*}$ - from DiGr into Gra as follows: for a directed loop free graph ( $Y$, $S)$ put

$$
(X, R, a, b) *(Y, S)=(V, T)
$$

where $V=X \times S / \sim$ where $\sim$ is the smallest equivalence such that

$$
\begin{aligned}
& \text { for } s=(x, y), t=(z, y) \in S \text { we have }(b, s) \sim(b, t) \\
& \text { for } s=(x, y), t=(y, z) \in S \text { we have }(b, s) \sim(a, t) \\
& \text { for } s=(x, y), t=(x, z) \in S \text { we have }(a, s) \sim(a, t) .
\end{aligned}
$$

Then for each $s \in S$ there is a one-to-one mapping $i_{s}: X \rightarrow V$ such that $i_{s}(x)$ is the class of $\sim$ containing $(x, s)$ for each $x \in X$. Put

$$
T=\left\{\left\{i_{s}(x), i_{s}(y)\right\} ;\{x, y\} \in R, s \in S\right\} .
$$


For a compatible mapping $f:(Y, S) \rightarrow\left(Y^{\prime}, S^{\prime}\right)$ define a compatible mapping

$$
(X, R, a, b) * f:(X, R, a, b) *(Y, S) \rightarrow(X, R, a, b) *\left(Y^{\prime}, S^{\prime}\right)
$$

such that for each $s \in S$ we have

$$
((X, R, a, b) * f) \cdot i_{s}=i_{s^{\prime}}
$$

where $s^{\prime} \in S^{\prime}$ such that

$$
s^{\prime}=(f(x), f(y)) \quad \text { whenever } s=(x, y) .
$$

A šíp $(X, R, a, b)$ is called a rigid šip if for every directed graph $(Y, S)$ and for every compatible mapping

$$
f:(X, R) \rightarrow(X, R, a, b) *(Y, S)
$$

there is $s \in S$ with $f=i_{s}$.

THEOREM 2.5. If $(X, R, a, b)$ is a rigid šip then $(X, R, a, b) *-$ is a full embedding.

Proof. See [12], [13].

By Condition d) of Corollary 2.3, if $a, b \in Z$ are distinct points then ( $Z$, $S, a, b)$ is a rigid šíp. Thus from Corollary 2.3 and Theorem 2.5 we obtain:

THEOREM 2.6. There is a full subcategory $\mathbf{C}$ of Gra such that

a) $\mathbf{C}$ is binding;

b) for every compatible mapping $f:(X, R) \rightarrow(Y, S)$ between objects of $\mathbf{C}$

b1) there is an infinite set $T$ of disjoint triangles in $(X, R)$ such that if $\{x$, $y, z\}$ and $\{u, v, w\}$ are distinct triangles in $T$ then

$$
\{f(x), f(y), f(z)\} \cap\{f(u), f(v), f(w)\}=\emptyset ;
$$

b2) for every $x \in X$ there is an infinite set $T_{x}$ of $X$ such that $f / T_{x}$ is one-to-one, if $t \in T_{x}$ then $\{x, t) \in R$ and for every pair $u, v \in T_{x}, u \neq v$ and for every $w \in Y$ either

$$
w=f(x) \quad \text { or } \quad\{w, f(u)\} \notin S \quad \text { or } \quad\{w, f(v)\} \notin S ;
$$

b3) for every edge $\{x, y\} \in R$ there is an infinite subset $T$ of $X$ such that $f / T$ is one-to-one and $\{x, t\},\{y, t\} \in R$ for each $t \in T$.

3. Lattice theoretical representations. The aim of this section is to prove that $\mathscr{A}(B) *$ and $\mathscr{A}(B)^{*}$ are binding varieties of bounded lattices. We prove this only for $\mathscr{A}(B)_{*}$, the proof for $\mathscr{A}(B)^{*}$ being dual. First we recall some useful facts:

Proposition 3.1. Every finite subdirectly irreducible lattice in $\mathscr{A}(B)_{*}$ is a 
sublattice of $I_{*}(\mathscr{G})$ for a finite complete bipartite graph $\mathscr{G}$. If $\mathscr{G}, \mathscr{G}^{\prime}$ are complete bipartite graphs, $\mathscr{G}$ a full subgraph of $\mathscr{G}^{\prime}$ and $f: \mathscr{G} \rightarrow \mathscr{G}^{\prime}$ the inclusion map, then $f$ can be extended to an embedding $f: I_{*}(\mathscr{G}) \rightarrow I_{*}\left(\mathscr{G}^{\prime}\right)$.

Proof. For the first fact see [3], and for the second one see [1].

Hence we immediately get:

Corollary 3.2. If $L$ is a finite lattice in $\mathscr{A}(B)_{*}$ and $x, y$ are distinct points of $L$ then for some finite complete bipartite graph $\mathscr{G}$ and $a$ homomorphism $f: L \rightarrow I_{*}(\mathscr{G}), f(x) \neq f(y)$ holds.

We describe a construction of a functor $\Phi$ from Gra to $\mathscr{A}(B)_{*}$.

Construction 3.3. Let $\mathscr{G}=(V, E)$ be a graph and $a \notin V \cup E$ a point. Let $\Theta_{\mathscr{G}}$ denote the smallest congruence of a free lattice $\mathscr{F}(V \cup E \cup\{a\})$ in $\mathscr{A}(B) *$ over $V \cup E \cup\{a\}$ such that

1) for each $v \in V \cup\{a\}$ and for each $e \in E$,

$$
v+e \Theta_{\mathscr{G}} 1
$$

2) for each edge $e=\{v, w\} \in E$,

$$
v \cdot w \cdot e \Theta_{\mathscr{G}} 0
$$

3) for each triangle $e_{1}=\{u, v\}, e_{2}=\{v, w\}, e_{3}=\{u, w\} \in E$,

$$
e_{1} \cdot e_{2} \cdot e_{3} \Theta_{\mathscr{G}} 0 \text { and } a \cdot v \cdot w \cdot u \Theta_{\mathscr{G}} 0 .
$$

Set

$$
\Phi(\mathscr{G})=\mathscr{F}(V \cup E \cup\{a\}) / \Theta_{\mathscr{G}}
$$

For a compatible mapping $f: \mathscr{G}=(V, E) \rightarrow \mathscr{H}=(W, F)$, let

$$
f^{\#}: \mathscr{F}(V \cup E \cup\{a\}) \rightarrow \mathscr{F}(W \cup F \cup\{a\})
$$

denote its free extension (assume that $a \notin W \cup F$ ) that is, $f^{\#}(v)=f(v)$ for each $v \in V, f^{\#}(\{v, w\})=\{f(v), f(w)\}$ for each $\{v, w\} \in E, f^{\#}(a)$ $=a$. Since $f$ is a compatible mapping we get that $f^{\#}$ maps $\Theta_{\mathscr{G}}$ into $\Theta_{\mathscr{H}}$ Thus there exists a homomorphism, denoted by $\Phi f$, from $\mathscr{F}(V \cup E \cup$ $\{a\}) / \Theta_{\mathscr{G}}$ to $\mathscr{F}(W \cup F \cup\{a\}) / \Theta_{\mathscr{H}}$ such that the following diagram commutes

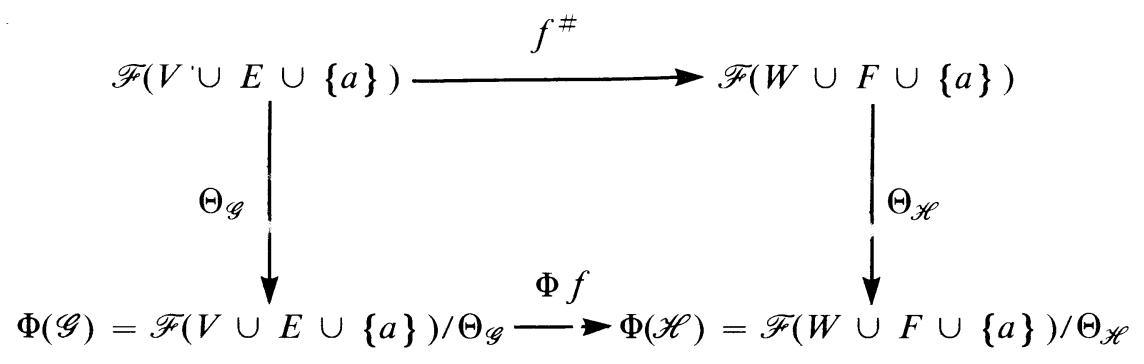


Since $V \cup E \cup\{a\} / \Theta_{\mathscr{G}}$ generates $\Phi(\mathscr{G})$ we get that $\Phi: \mathrm{Gra} \rightarrow \mathscr{A}(B)_{*}$ is a functor.

LEMMA 3.4. If $U$ (or $V$ ) is the natural forgetful functor from Gra (or $\mathscr{A}(B)_{*}$, resp. ) to Set then there is a monotransformation from $U$ to $V \circ \Phi$, in particular $\Phi$ is faithful. For each graph $\mathscr{G}=(V, E)$, if $x+y=1$ for some $x$, $y \in \Phi(\mathscr{G}), x \neq 1 \neq y$ then there are $v \in V \cup\{a\}$ and $e \in E$ with either $v$ $\leqq x$ and $e \leqq y$ or $v \leqq y$ and $e \leqq x$.

Proof. For a graph $\mathscr{G}=(V, E)$ define

$$
\mathscr{B}(\mathscr{G})=(V \cup\{a\} \cup E, S)
$$

where

$$
S=\{\{v, e\} ; v \in V \cup\{a\}, e \in E\} .
$$

Obviously $\mathscr{B}(\mathscr{G})$ is a complete bipartite graph. It is easy to see that $I_{*}(\mathscr{B}(\mathscr{G}))$ fulfils 1$), 2$ ) and 3 ), thus there is

$$
\psi: \Phi(\mathscr{G}) \rightarrow I_{*}(\mathscr{B}(\mathscr{G}))
$$

such that $\psi$ maps the class of $\Theta_{\mathscr{G}}$ containing $x \in V \cup E \cup\{a\}$ onto $x$. Hence we get the first statement. Proposition 1.4 in [1] immediately implies the second one.

In what follows, we shall identify a point $x \in V \cup E \cup\{a\}$ with the class of $\Theta_{\mathscr{G}}$ containing $x$; thus $V \cup E \cup\{a\} \subset \Phi(\mathscr{G})$.

Lemma 3.5. Let $A$ be a subset of $V \cup E \cup\{a\}$ such that either $|A \cap V|$ $\leqq 1$ or $|A \cap E| \leqq 1$. Then $\Pi A=0$ if and only if one of the following occurs:

a) there is a triangle $e_{1}=\{u, v\}, e_{2}=\{u, w\}, e_{3}=\{v, w\} \in E$ with $e_{1}$, $e_{2}, e_{3} \in A$;

b) there is a triangle $e_{1}=\{u, v\}, e_{2}=\{u, w\}, e_{3}=\{v, w\} \in E$ with $a, u$, $v, w \in A$;

c) there is an edge $e=\{u, v\} \in E$ with $e, u, v \in A$.

Proof. Assume $|A \cap V| \leqq 1$ and define

$$
\varphi: V \cup E \cup\{a\} \rightarrow I_{*}(\mathscr{B},(\mathscr{G}))
$$

as follows:

$$
\begin{aligned}
& \varphi(v)=v \quad \text { for } v \in(V \cup E \cup\{a\})-A, \\
& \varphi(v)=1 \quad \text { for } v \in A \cap(V \cup\{a\}),
\end{aligned}
$$

choose $e_{0} \in A \cap E$ and set $\varphi(e)=e_{0}$ for $e \in A \cap E$. If $A$ fails a), b) and c) then $\varphi$ can be extended to a homomorphism

$$
\bar{\varphi}: \Phi(\mathscr{G}) \rightarrow I_{*}(\mathscr{B}(\mathscr{G}))
$$


and $\Pi \bar{\varphi}(A)=e_{0}$; thus $\Pi(A) \neq 0$. If $|A \cap E| \leqq 1$, define

$$
\varphi: V \cup E \cup\{a\} \rightarrow I_{*}(\mathscr{B}(\mathscr{G}))
$$

as follows:

$$
\begin{array}{ll}
\varphi(v)=v & \text { for } v \in(V \cup E \cup\{a\})-A, \\
\varphi(e)=1 & \text { for } e \in A \cap E,
\end{array}
$$$$
\text { choose } v_{0} \in A \cap(V \cup\{a\}) \text { and put }
$$

$$
\varphi(v)=v_{0} \quad \text { for } v \in A \cap(V \cup\{a\}) .
$$

If $A$ does not satisfy a), b) and c) then $\varphi$ can be extended to a homomorphism

$$
\bar{\varphi}: \Phi(\mathscr{G}) \rightarrow I_{*}(\mathscr{B}(\mathscr{G}))
$$

thus $\prod(A) \neq 0$. On the other hand, if $A$ fulfills a) or b) or c) then by the definition of $\Theta_{\mathscr{G}}$ we get $\Pi A=0$.

The following lemmas enable us to distinguish elements of $X \cup R \cup$ $\{a\}$ amongst those of $\Phi(\mathscr{G})$.

Lemma 3.6. Let $\mathscr{G}=(X, R)$ be a graph such that for each point $x \in X$ an infinite subset $E_{x}$ of $R$ is given such that:

a) for each $e=\{u, v\} \in E_{x}$ we have either $x=u$ or $x=v$;

b) if $\{x, u\},\{x, v\} \in E_{x}$ then for every vertex $y \in X-\{x\}$ either $\{y, u\}$ $\notin R$ or $\{y, v\} \notin R$.

Then for each $x \in X$ and each $b \in \Phi(\mathscr{G})$ with $b>x$ there exists an edge $e$ $=\{x, u\} \in E_{x}$ with $b \cdot e \cdot u \neq 0$.

Proof. Assume that $b>x \in X$. Then there is a finite subset $A \subset X \cup R$ $\cup\{a\}$ such that

A) $a, x \in A$ and $b$ lies in the sublattice $L$ of $\Phi(\mathscr{G})$ generated by $A$;

B) for every edge $e=\{u, v\} \in R, e \in A$ if and only $u, v \in A$;

C) for every triangle $e_{1}=\{u, v\}, e_{2}=\{u, w\}, e_{3}=\{v, w\} \in R$ if $\mid\left\{e_{1}\right.$, $\left.e_{2}, e_{3}\right\} \cap A \mid \geqq 2$ then $e_{1}, e_{2}, e_{3} \in A$;

D) there is a triangle $e_{1}=\{u, v\}, e_{2}=\{u, w\}, e_{3}=\{v, w\} \in R$ with $e_{1}$, $e_{2}, e_{3} \in A$.

Indeed, there is a polynomial $p$ such that

$$
p\left(c_{1}, c_{2}, \ldots, c_{n}\right)=b \text { and } c_{1}, c_{2}, \ldots, c_{n} \in X \cup R \cup\{a\} .
$$

Then there is a finite subset $A^{\prime}$ of $X$ such that

$$
c_{1}, c_{2}, \ldots, c_{n} \in A^{\prime} \cup R^{\prime} \cup\{a\}
$$

for the full subgraph $\left(A^{\prime}, R^{\prime}\right)$ of $(X, R)$. Choose a triangle $e_{1}=\{u, v\}, e_{2}=$ $\{u, w\}, e_{3}=\{v, w\} \in R$ and put

$$
\bar{A}=A^{\prime} \cup\{x, u, v, w\} .
$$


If $(\bar{A}, \bar{R})$ is the full subgraph of $(X, R)$ determined by $\bar{A}$ then $A=\bar{A} \cup \bar{R}$ $\cup\{a\}$ has the required properties. By Corollary 3.2 there is a finite complete bipartite graph $(V, E)$ (with a decomposition $V_{1}, V_{2}$ of $V$ with $E$ $\left.=\left\{\{v, w\} ; v \in V_{1}, w \in V_{2}\right\}\right)$ such that there is a homomorphism

$$
f: L \rightarrow I_{*}(V, E) \text { with } f(b) \neq f(x) .
$$

Since $b>x$ we obtain $f(b)>f(x)$, and thus $f(x) \neq 1$. Now for every $e \in$ $A \cap R$,

$$
f(x)+f(e)=1,
$$

thus $f(e) \neq 0$. If $f(v)=0$ for some $v \in A \cap(X \cup\{a\})$ then $f(e)=1$ for each $e \in A \cap R$ because $f(v)+f(e)=1$, but this is a contradiction with D) because

$$
f\left(e_{1}\right) \cdot f\left(e_{2}\right) \cdot f\left(e_{3}\right)=0 .
$$

Thus we conclude:

E) $f(x) \neq 0,1$ and for every $c \in A, f(c)>0$.

Assume that $f(x)=B_{x} \subset V_{1}$. Since $f(v)+f(e)=1$ for every $v \in A \cap$ $(X \cup\{a\})$ and $e \in A \cap R$, we get that $f(e)=B_{e} \subset V_{2}$ or $=1$ for every $e$ $\in A \cap R$ and $f(v)=B_{v} \subset V_{1}$ or $=1$ for every $v \in A \cap(X \cup\{a\})$. Furthermore,

F) for every triangle $e_{1}^{\prime}=\left\{u^{\prime}, v^{\prime}\right\}, e_{2}^{\prime}=\left\{u^{\prime}, w^{\prime}\right\}, e_{3}^{\prime}=\left\{v^{\prime}, w^{\prime}\right\} \in R$ either

$$
\begin{aligned}
& u^{\prime}, v^{\prime}, w^{\prime} \in A \text { or } \\
& \left|\left\{u^{\prime}, v^{\prime}, w^{\prime}\right\} \cap A\right| \leqq 1 \text { or } \\
& \prod f\left(A \cap\left\{a, u^{\prime}, v^{\prime}, w^{\prime}\right\}\right) \neq 1 .
\end{aligned}
$$

Indeed, assume that $u^{\prime}, v^{\prime} \in A$, then $e_{1}^{\prime} \in A$ by B) and

$$
f\left(e_{1}^{\prime}\right) \cdot f\left(u^{\prime}\right) \cdot f\left(v^{\prime}\right)=0 .
$$

Since $f\left(e_{1}^{\prime}\right) \neq 0$, we get that $f\left(u^{\prime}\right) \cdot f\left(v^{\prime}\right) \neq 1$. By the hypothesis, there is an edge $e_{0}=\{x, y\} \in E_{x}$ such that $\{y, t\} \in R$ for $t \in A$ implies $t=x$. Define a graph

$$
\mathscr{H}=\left(V \cup X \cup R, E^{\prime}\right)
$$

where

$$
\begin{aligned}
E^{\prime} & =E \cup\{\{z, r\} ; z \in X, r \in R\} \\
& \cup\left\{\{z, v\} ; z \in X, v \in V_{2}\right\} \\
& \cup\left\{\{r, v\} ; r \in R, v \in V_{1}\right\} .
\end{aligned}
$$

Since $(V, E)$ is a complete bipartite graph with the decomposition $V_{1}, V_{2}$, we get that $\mathscr{H}$ is a complete bipartite graph. By Proposition 3.1 there is a canonical embedding 


$$
g: I_{*}(V, E) \rightarrow I_{*}(\mathscr{H})
$$

such that $g / V$ is the inclusion of $(V, E)$ into $\mathscr{H}$. Define

$$
\varphi: X \cup R \cup\{a\} \rightarrow I_{*}(\mathscr{H})
$$

as follows:

$$
\begin{aligned}
& \varphi(z)=g \circ f(z) \text { for } z \in A \\
& \varphi(z)=z \text { for } z \in X \cup R-\left(A \cup\left\{e_{0}, y\right\}\right) \\
& \varphi\left(e_{0}\right)=1,
\end{aligned}
$$

and choose $c \in V_{1} \cup\{y\}$ with $c \notin B_{x}$ and $\{c\}<g_{\circ} f(b)$ (it is possible because $f(b)>f(x))$ and put $\varphi(y)=c$.

Clearly $\varphi(v)+\varphi(e)=1$ in $I_{*}(\mathscr{H})$ for every $v \in X \cup\{a\}, e \in R$. The edge $e_{0}$ satisfies

$$
\varphi(x) \cdot \varphi(y) \cdot \varphi\left(e_{0}\right)=B_{x} \cap\{c\} .
$$

For $e=\{z, t\} \in R, e \neq e_{0}$, by B) either $e, z, t \in A$ then

$$
\varphi(e) \cdot \varphi(z) \cdot \varphi(t)=g \circ f(e) \cdot g \circ f(z) \cdot g \circ f(t)=0
$$

or $|\{e, z, t\} \cap A| \leqq 1$ and then

$$
\varphi(e) \cdot \varphi(z) \cdot \varphi(t)=0 .
$$

Thus

G) for each edge $e=\{z, t\} \in R$,

$$
\varphi(e) \cdot \varphi(z) \cdot \varphi(t)=0 .
$$

If $e_{1}^{\prime}=\left\{u^{\prime}, v^{\prime}\right\}, e_{2}^{\prime}=\left\{u^{\prime}, w^{\prime}\right\}, e_{3}^{\prime}=\left\{v^{\prime}, w^{\prime}\right\} \in R$ is a triangle then by C) either $e_{1}^{\prime}, e_{2}^{\prime}, e_{3}^{\prime} \in A$ and thus

$$
\varphi\left(e_{1}^{\prime}\right) \cdot \varphi\left(e_{2}^{\prime}\right) \cdot \varphi\left(e_{3}^{\prime}\right)=g \circ f\left(e_{1}^{\prime}\right) \cdot g \circ f\left(e_{2}^{\prime}\right) \cdot g \circ f\left(e_{3}^{\prime}\right)=0
$$

or $\left|\left\{e_{1}^{\prime}, e_{2}^{\prime}, e_{3}^{\prime}\right\} \cap A\right| \leqq 1$ and then

$$
\varphi\left(e_{1}^{\prime}\right) \cdot \varphi\left(e_{2}^{\prime}\right) \cdot \varphi\left(e_{3}^{\prime}\right)=0
$$

because $e_{0} \in\left\{e_{1}^{\prime}, e_{2}^{\prime}, e_{3}^{\prime}\right\}$ implies $\left\{e_{1}^{\prime}, e_{2}^{\prime}, e_{3}^{\prime}\right\} \cap A=\emptyset$. Moreover by $\mathrm{F}$ ) if $u^{\prime}, v^{\prime}, w^{\prime} \in A$ then by A)

$$
\begin{aligned}
\varphi(a) \cdot \varphi\left(u^{\prime}\right) \cdot \varphi\left(v^{\prime}\right) \cdot & \varphi\left(w^{\prime}\right) \\
& =g \circ f(a) \cdot g \circ f\left(u^{\prime}\right) \cdot g \circ f\left(v^{\prime}\right) \cdot g \circ f\left(w^{\prime}\right)=0 .
\end{aligned}
$$

If $\left|\left\{u^{\prime}, v^{\prime}, w^{\prime}\right\} \cap A\right| \leqq 1$ then

$$
\varphi(a) \cdot \varphi\left(u^{\prime}\right) \cdot \varphi\left(v^{\prime}\right) \cdot \varphi\left(w^{\prime}\right)=0 .
$$

If $\left|\left\{u^{\prime}, v^{\prime}, w^{\prime}\right\} \cap A\right|=2$ then

$$
\Pi \varphi\left(A \cap\left\{a, u^{\prime}, v^{\prime}, w^{\prime}\right\}\right) \neq 1
$$

hence 


$$
\varphi(a) \cdot \varphi\left(u^{\prime}\right) \cdot \varphi\left(v^{\prime}\right) \cdot \varphi\left(w^{\prime}\right)=0
$$

(because if e.g. $w^{\prime} \notin A$ then by the assumption on $y, w^{\prime} \neq y$ ). Thus

$\mathrm{H})$ for each triangle $e_{1}^{\prime}=\left\{u^{\prime}, v^{\prime}\right\}, e_{2}^{\prime}=\left\{u^{\prime}, w^{\prime}\right\}, e_{3}^{\prime}=\left\{v^{\prime}, w^{\prime}\right\} \in R$,

$$
\begin{aligned}
& \varphi\left(e_{1}^{\prime}\right) \cdot \varphi\left(e_{2}^{\prime}\right) \cdot \varphi\left(e_{3}^{\prime}\right)=0 \quad \text { and } \\
& \varphi(a) \cdot \varphi\left(u^{\prime}\right) \cdot \varphi\left(v^{\prime}\right) \cdot \varphi\left(w^{\prime}\right)=0 .
\end{aligned}
$$

Hence $\varphi$ can be extended to a homomorphism

$$
\varphi^{*}: \Phi(\mathscr{G}) \rightarrow I_{*}(\mathscr{H}) .
$$

Then $\varphi^{*}\left(b \cdot y \cdot e_{0}\right)=\{c\} \neq 0$; thus $b \cdot y \cdot e_{0} \neq 0$.

LEMMA 3.7. Let $(X, R)$ be a graph such that for each edge $e=\{x, y\} \in R$ an infinite subset $X_{e}$ of $X$ is given such that for each $z \in X_{e},\{x, y, z\}$ is a triangle. Then for each edge $e=\{x, y\} \in R$ and for each $b \in \Phi(X, R)$ with $b>$ e there is $z \in X_{e}$ such that

$$
b \cdot\{x, z\} \cdot\{y, z\} \neq 0 .
$$

Proof. Assume that $b>e^{\prime}=\left\{x^{\prime}, y^{\prime}\right\} \in R$. Analogously as in the proof of Lemma 3.6 we can choose a finite subset $A$ of $X \cup R \cup\{a\}$ satisfying the four conditions below:

A) $a, e^{\prime} \in A$ and $b \in L$ where $L$ is the sublattice of $\Phi(X, R)$ generated by $A$;

B) for every edge $e=\{u, v\} \in R, e \in A$ if and only if $u, v \in A$;

C) for every triangle $e_{1}=\{u, v\}, e_{2}=\{u, w\}, e_{3}=\{v, w\} \in R$ with

$$
\left|\left\{e_{1}, e_{2}, e_{3}\right\} \cap A\right| \geqq 2
$$

we have $e_{1}, e_{2}, e_{3} \in A$;

D) there is a triangle $e_{1}=\{u, v\}, e_{2}=\{u, w\}, e_{3}=\{v, w\} \in R$ with $e_{1}$, $e_{2}, e_{3} \in A$.

By Corollary 3.2 there are a complete bipartite graph $(V, E)$ (with a decomposition of $V_{1}, V_{2}$ of $V$ with $\left.E=\left\{\left\{v_{1}, v_{2}\right\} ; v_{1} \in V_{1}, v_{2} \in V_{2}\right\}\right)$ and a homomorphism $f: L \rightarrow I_{*}(V, E)$ with $f(b) \neq f\left(e^{\prime}\right)$. Then $f(b)>f\left(e^{\prime}\right)$ and hence $1 \neq f\left(e^{\prime}\right)$. Since

$$
f(v)+f\left(e^{\prime}\right)=1 \text { for each } v \in A \cap(X \cup\{a\})
$$

we get that $f(v) \neq 0$. If $f(e)=0$ for some $e \in A \cap R$ then $f(v)=1$ for each $v \in X \cup\{a\}$, but this is a contradiction with D) and thus

E) $f(v) \neq 0$ for each $v \in A$ and $f\left(e^{\prime}\right) \neq 1$.

Assume that $f\left(e^{\prime}\right)=B_{e^{\prime}} \subset V_{1}$, then $f(e)=B_{e} \subset V_{1}$ or $=1$ for each $e$ $\in A \cap R$ and $f(v)=B_{v} \subset V_{2}$ or $=1$ for each $v \in A \cap(X \cup\{a\})$. Clearly,

F) for each triangle $e_{1}=\{u, v\}, e_{2}=\{u, w\}, e_{3}=\{v, w\} \in R$ either 


$$
\begin{aligned}
& \{u, v, w\} \subset A, \text { or } \\
& |\{u, v, w\} \cap A| \leqq 1 \text { or } \\
& \prod f(A \cap\{a, u, v, w\}) \neq 1 .
\end{aligned}
$$

By the hypothesis, there is $z \in E_{e^{\prime}}-A$, i.e.,

$$
\left\{x^{\prime}, z\right\}=e_{1}^{\prime}, \quad\left\{y^{\prime}, z\right\}=e_{2}^{\prime} \in R-A .
$$

Put $\mathscr{H}=\left(V \cup X \cup R, E^{\prime}\right)$ where

$$
\begin{aligned}
& E^{\prime}=E \cup\left\{\{v, x\} ; v \in V_{1}, x \in X\right\} \cup\left\{\{v, r\} ; v \in V_{2}, r \in R\right\} \\
& \cup\{\{x, r\} ; x \in X, r \in R\} .
\end{aligned}
$$

Obviously $\mathscr{H}$ is a complete bipartite graph and there is a homomorphism

$$
g: I_{*}(V, E) \rightarrow I_{*}(\mathscr{H})
$$

such that $g / V$ is the inclusion mapping. Further there is $c \in V_{1} \cup\left\{e_{1}^{\prime}\right\}$ such that $c \notin B_{e^{\prime}}$ and $\{c\}<g_{\circ} f(b)$. Define a mapping

$$
\varphi: X \cup R \cup\{a\} \rightarrow I_{*}(\mathscr{H})
$$

by

$$
\begin{aligned}
& \varphi(z)=g \circ f(z) \text { for } z \in A \\
& \varphi(z)=z \text { for } z \in X \cup R-\left(A \cup\left\{e_{1}^{\prime}, e_{2}^{\prime}\right\}\right) \\
& \varphi\left(e_{1}^{\prime \prime}\right)=\varphi\left(e_{2}^{\prime}\right)=c .
\end{aligned}
$$

It is easy to see that $\varphi(v)+\varphi(e)=1$ for each $e \in R, v \in X \cup\{a\}$. For $e$ $=\{x, y\} \in R$ either

$$
e, x, y \in A \text { or }|\{e, x, y\} \cap A| \leqq 1 ;
$$

in both cases

$$
\varphi(e) \cdot \varphi(x) \cdot \varphi(y)=0 .
$$

For a triangle $e_{1}=\{u, v\}, e_{2}=\{u, w\}, e_{3}=\{v, w\} \in R$ either $e_{1}, e_{2}, e_{3} \in$ $A$ and

$$
\boldsymbol{\varphi}\left(e_{1}\right) \cdot \boldsymbol{\varphi}\left(e_{2}\right) \cdot \boldsymbol{\varphi}\left(e_{3}\right)=g \circ f\left(e_{1}\right) \cdot g \circ f\left(e_{2}\right) \cdot g \circ f\left(e_{3}\right)=0
$$

follows, or $\left\{e_{1}, e_{2}, e_{3}\right\}=\left\{e^{\prime}, e_{1}^{\prime}, e_{2}^{\prime}\right\}$ so that

$$
\varphi\left(e_{1}\right) \cdot \varphi\left(e_{2}\right) \cdot \varphi\left(e_{3}\right)=B_{e^{\prime}} \cap\{c\}=0,
$$

or $\left|\left\{e_{1}, e_{2}, e_{3}^{\prime}\right\} \cap A\right| \leqq 1$ and $\left\{e_{1}, e_{2}, e_{3}\right\} \neq\left\{e^{\prime}, e_{1}^{\prime}, e_{2}^{\prime}\right\}$; then obviously

$$
\boldsymbol{\varphi}\left(e_{1}\right) \cdot \boldsymbol{\varphi}\left(e_{2}\right) \cdot \boldsymbol{\varphi}\left(e_{3}\right)=0 .
$$

Furthermore, if $u, v, w \in A$ then

$$
\begin{aligned}
\varphi(u) \cdot \varphi(v) \cdot \varphi(w) \cdot \varphi(a) & =g \circ f(u) \cdot g \circ f(v) \cdot g \circ f(w) \cdot g \circ f(a) \\
& =0
\end{aligned}
$$


if $|\{u, v, w\} \cap A| \leqq 1$ then again we have

$$
\varphi(a) \cdot \varphi(u) \cdot \varphi(v) \cdot \varphi(w)=0 .
$$

If $|\{u, v, w\} \cap A|=2$ then

$$
\Pi \varphi(A \cap\{a, u, v, w\})=\prod g \circ f(A \cap\{a, u, v, w\}) \neq 1
$$

and hence

$$
\varphi(a) \cdot \varphi(u) \cdot \varphi(v) \cdot \varphi(w)=0 ;
$$

(if e.g. $w \notin A$, then $w \notin \Pi \varphi(A \cap\{a, u, v, w\}))$. Thus $\varphi$ can be extended to a homomorphism.

$$
\varphi^{*}: \Phi(X, R) \rightarrow I_{*}(\mathscr{H})
$$

with

$$
\varphi^{*}(b) \cdot \varphi^{*}\left(e_{1}^{\prime}\right) \cdot \varphi^{*}\left(e_{2}^{\prime}\right)=g \circ f(b) \cdot\{c\}=\{c\} \neq 0
$$

thus $b \cdot e_{1}^{\prime} \cdot \mathrm{e}_{2}^{\prime} \neq 0$.

LeMmA 3.8. Let $\mathscr{G}=(X, R)$ be a graph with a given infinite set $T$ of disjoint triangles in $(X, R)$. Then for every $b \in \Phi(\mathscr{G})$ with $b>$ a there is $a$ triangle $\{u, v, w\}$ with $b \cdot u \cdot v \cdot w \neq 0$.

Proof. Assume that $b>a$. Analogously to the proof of Lemma 3.6 there is a finite subset $A$ of $X \cup R \cup\{a\}$ such that

A) $a \in A$ and $b \in L$ where $L$ is a sublattice of $\Phi(\mathscr{G})$ generated by $A$;

B) for every edge $e=\{x, y\} \in R, e \in A$ if and only if $x, y \in A$;

C) for triangles $e_{1}=\{u, v\}, e_{2}=\{u, w\}, e_{3}=\{v, w\} \in R$, if

$$
\left|\left\{e_{1}, e_{2}, e_{3}\right\} \cap A\right| \geqq 2
$$

then $e_{1}, e_{2}, e_{3} \in A$;

D) there is a triangle $e_{1}=\{u, v\}, e_{2}=\{u, w\}, e_{3}=\{v, w\} \in R$ with $\left\{e_{1}, e_{2}, e_{3}\right\} \subset A$.

By Corollary 3.2 there are a complete bipartite graph $(V, E)$ (with a decomposition of $V_{1}, V_{2}$ of $V$ with $E=\left\{\left\{v_{1}, v_{2}\right\} ; v_{1} \in V_{1}, v_{2} \in V_{2}\right\}$ ), and a homomorphism $f: L \rightarrow I_{*}(V, E)$ with $f(a) \neq f(b)$. By the same arguments as in Lemma 3.6 we obtain:

E) $f(c) \neq 0$ for each $c \in A, f(a) \neq 1$;

F) for each triangle $\{u, v, w\}$ either

$$
\begin{aligned}
& u, v, w \in A \text { or } \\
& |\{u, v, w\} \cap A| \leqq 1 \text { or } \\
& \prod f(A \cap\{a, u, v, w\}) \neq 1 .
\end{aligned}
$$

Assume that $f(a)=B_{a} \subset V_{1}$, then $f(v)=B_{v} \subset V_{1}$ or $=1$ for each $v \in$ $A \cap X, f(e)=B_{e} \subset V_{2}$ or $=1$ for each $e \in A \cap R$. Put 


$$
\mathscr{H}=\left(V \cup X \cup R, E^{\prime}\right)
$$

where

$$
\begin{aligned}
& E^{\prime}=E \cup\{\{x, r\} ; x \in X, r \in R\} \cup\left\{\{v, x\} ; v \in V_{2}, x \in X\right\} \\
& \cup\left\{\{v, r\} ; v \in V_{1}, r \in R\right\} .
\end{aligned}
$$

Obviously $\mathscr{H}$ is a complete bipartite graph and there is a homomorphism

$$
g: I_{*}(V, E) \rightarrow I_{*}(\mathscr{H})
$$

such that $g / V$ is the inclusion. Choose $c \in V_{1}$ such that $c \notin B_{a}$ and $\{c\}<$ $f(b)$. Choose a triangle $\left\{u^{\prime}, v^{\prime}, w^{\prime}\right\}$ such that

$$
\left\{u^{\prime}, v^{\prime}, w^{\prime}\right\} \cap A=\emptyset
$$

(this is possible by the hypothesis). Define a mapping

$$
\varphi: X \cup R \cup\{a\} \rightarrow I_{*}(\mathscr{H})
$$

as follows:

$$
\begin{aligned}
& \varphi(z)=g \circ f(z) \text { for } z \in A \\
& \varphi(z)=z \quad \text { for } z \in X \cup R-\left(A \cup\left\{u^{\prime}, v^{\prime}, w^{\prime}\right\}\right) \\
& \varphi\left(u^{\prime}\right)=\varphi\left(v^{\prime}\right)=\varphi\left(w^{\prime}\right)=c .
\end{aligned}
$$

It is clear that $\varphi(v)+\varphi(e)=1$ for each $v \in X \cup\{a\}, e \in R$. Similarly to Lemma 3.6 we conclude that if $e_{1}=\{u, v\}, e_{2}=\{u, w\}, e_{3}=\{v, w\} \in R$ is a triangle then

$$
\begin{aligned}
& \varphi\left(e_{1}\right) \cdot \varphi\left(e_{2}\right) \cdot \varphi\left(e_{3}\right)=0 \text { and } \\
& \varphi(a) \cdot \varphi(u) \cdot \varphi(v) \cdot \varphi(w)=0 .
\end{aligned}
$$

If $e=\{x, y\}$ then for $e \notin T-\left\{\left\{u^{\prime}, v^{\prime}\right\},\left\{u^{\prime}, w^{\prime}\right\},\left\{v^{\prime}, w^{\prime}\right\}\right\}$ evidently

$$
\varphi(e) \cdot \varphi(x) \cdot \varphi(y)=0 .
$$

If $e \in\left\{\left\{u^{\prime}, v^{\prime}\right\},\left\{u^{\prime}, w^{\prime}\right\},\left\{v^{\prime}, w^{\prime}\right\}\right\}$ then $e \notin A$ and hence

$$
\varphi(e) \cdot \varphi(x) \cdot \varphi(y)=0 .
$$

Therefore there exists a homomorphism

$$
\varphi^{*}: \Phi(\mathscr{G}) \rightarrow I_{*}(\mathscr{H})
$$

extending $\varphi$; thus

$$
\varphi^{*}(b) \cdot \varphi^{*}\left(u^{\prime}\right) \cdot \varphi^{*}\left(v^{\prime}\right) \cdot \varphi^{*}\left(w^{\prime}\right)=g \circ f(b) \cdot\{c\}=\{c\} \neq 0,
$$

but $b \cdot u^{\prime} \cdot v^{\prime} \cdot w^{\prime} \neq 0$ now follows.

THEOREM 3.9. Varieties $\mathscr{A}(B)_{*}$ and $\mathscr{A}(B)^{*}$ are binding. 
Proof. Let $\varphi: \Phi(\mathscr{G}) \rightarrow \Phi(\mathscr{H})$ be a homomorphism where $\mathscr{G}=(X, R), \mathscr{H}$ $=(Y, S)$. Then for each $v \in X \cup\{A\}, e \in R$,

$$
\varphi(v)+\varphi(e)=1 ;
$$

thus if $\varphi(e)=0$ for some $e \in R$ then $\varphi(v)=1$ for each $v \in X \cup\{a\}$. If $\mathscr{G}$ contains a triangle $e_{1}=\{x, y\}, e_{2}=\{x, z\}, e_{3}=\{y, z\} \in R$, then

$$
\varphi(a) \cdot \varphi(x) \cdot \varphi(y) \cdot \varphi(z)=0 ;
$$

this is a contradiction; analogously, if $\varphi(v)=0$ for some $v \in X$ then $\varphi(e)$ $=1$ for each $e \in R$ but

$$
\varphi\left(e_{1}\right) \cdot \varphi\left(e_{2}\right) \cdot \varphi\left(e_{3}\right)=0,
$$

a contradiction. Hence

A) If $\mathscr{G}$ contains a triangle then

$$
0 \notin \varphi(X \cup R \cup\{a\}) .
$$

Consider that for each edge $e_{1}=\{u, v\} \in R$ there is a vertex $w$ such that $e_{2}=\{u, w\}, e_{3}=\{v, w\} \in R$; i.e., each edge is in a triangle. Since

$$
\varphi\left(e_{1}\right) \cdot \varphi\left(e_{2}\right) \cdot \varphi\left(e_{3}\right)=\varphi\left(e_{1} \cdot e_{2} \cdot e_{3}\right)=0
$$

we see that $\varphi\left(e_{i}\right)=1$ for at most one $e_{i}$. Assume that $\varphi\left(e_{1}\right)=1$. Then $\varphi(u)$ $\neq 1 \neq \varphi(v)$ because

$$
\varphi\left(e_{1}\right) \cdot \varphi(u) \cdot \varphi(v)=\varphi\left(e_{1} \cdot u \cdot v\right)=\varphi(0)=0 .
$$

By Lemma 3.4 there are $x_{1}, y_{1}, x_{2}, y_{2} \in Y \cup S \cup\{a\}$ such that

$$
\begin{aligned}
& \varphi\left(e_{2}\right) \geqq x_{1}, \varphi(u) \geqq x_{2}, \varphi\left(e_{3}\right) \geqq y_{1}, \varphi(v) \geqq y_{2} \quad \text { and } \\
& x_{1}+x_{2}=1=y_{1}+y_{2}
\end{aligned}
$$

(because $\varphi\left(e_{2}\right)+\varphi(u)=1=\varphi\left(e_{3}\right)+\varphi(v)$ and $\left.\varphi\left(e_{2}\right), \varphi\left(e_{3}\right), \varphi(u), \varphi(v) \neq 1\right)$. Then

$$
0=\varphi\left(e_{1} \cdot u \cdot v\right)=\varphi\left(e_{1}\right) \cdot \varphi(u) \cdot \varphi(v) \geqq 1 \cdot x_{2} \cdot y_{2}=x_{2} \cdot y_{2} \neq 0
$$

by Lemma 3.5 . Hence $\varphi\left(e_{1}\right) \neq 1$. Thus we get $1 \notin \varphi(R)$. Since

$$
\varphi(u) \cdot \varphi(v) \cdot \varphi\left(e_{1}\right)=\varphi\left(u \cdot v \cdot e_{1}\right)=0
$$

we get that either $\varphi(u) \neq 1$ or $\varphi(v) \neq 1$. Assume that $\varphi(u)=1$, then

$$
1=\varphi\left(e_{1}+v\right)=\varphi\left(e_{1}\right)+\varphi(v)
$$

and by Lemma 3.4 there are $x, y \in Y \cup S \cup\{a\}$ with $\varphi\left(e_{1}\right) \geqq x, \varphi(v) \geqq y$ and $x+y=1$. This contradicts Lemma 3.5 because

$$
0=\varphi\left(e_{1} \cdot u \cdot v\right) \geqq 1 \cdot x \cdot y \neq 0 .
$$

\section{Therefore}

B) if $\mathscr{G}$ has no isolated vertices and each edge of $\mathscr{G}$ is in a triangle then 1 $\notin \varphi(X \cup R)$. 
Now for each edge $e=\{u, v\} \in R$ there are $x_{1}, y_{1}, x_{2}, y_{2} \in Y \cup S \cup$ $\{a\}$ with

$$
\begin{aligned}
& \varphi(u) \geqq x_{1}, \varphi(e) \geqq x_{2}, \varphi(v) \geqq y_{1}, \varphi(e) \geqq y_{2} \quad \text { and } \\
& x_{1}+x_{2}=1=y_{1}+y_{2}
\end{aligned}
$$

(we use Lemma 3.4). Then

$$
\begin{aligned}
0 & =\varphi(u \cdot e \cdot v)=\varphi(u) \cdot \varphi(e) \cdot \varphi(v) \geqq x_{1} \cdot y_{1}\left(x_{2}+y_{2}\right) \\
& \geqq x_{1} \cdot y_{1} \cdot x_{2}+x_{1} \cdot y_{1} \cdot y_{2} .
\end{aligned}
$$

From Lemma 3.5 it follows that for each pair $x, y \in Y \cup S \cup\{a\}$ there is at most one $z \in Y \cup S \cup\{a\}$ with $x \cdot y \cdot z=0$; hence $x_{2}=y_{2}$. An identical argument shows that if $\varphi(u) \geqq x_{3}$ then $x_{1}=x_{3}$, because

$$
\begin{aligned}
0=\varphi(u) \cdot \varphi(v) \cdot \varphi(e) \geqq\left(x_{1}+x_{3}\right) \cdot y_{1} \cdot x_{2} & \\
& \geqq x_{1} \cdot y_{1} \cdot x_{2}+x_{3} \cdot y_{1} \cdot x_{2} .
\end{aligned}
$$

Thus for each $z \in X \cup R$ there is at most one $u \in Y \cup S \cup\{a\}$ with $\varphi(z)$ $\geqq u$; put $\psi(z)=u$. If $e=\{u, v\} \in R$ then either $\psi(e) \in S$ and $\psi(u), \psi(v)$ $\in Y \cup\{a\}$ or $\psi(e) \in Y \cup\{a\}, \psi(u), \psi(v) \in S$. Since

$$
0=\varphi(u \cdot v \cdot e) \geqq \psi(e) \cdot \psi(u) \cdot \psi(v),
$$

Lemma 3.5 shows that the second case is impossible. Thus $\psi(e) \in S$ and $\psi(u), \psi(v) \in Y \cup\{a\}$; moreover, by Lemma 3.5,

$$
\psi(e)=\{\psi(u), \psi(v)\} .
$$

Furthermore, $\varphi(a)+\varphi(e)=1$ for every $e \in R$; hence either

$$
\varphi(a)=1 \text { or } \varphi(a) \geqq y \in Y \cup\{a\} .
$$

If $\{u, v, w\}$ is a triangle then

$$
0=\varphi(a) \cdot \varphi(u) \cdot \varphi(v) \cdot \varphi(w) \geqq \psi(a) \cdot \psi(u) \cdot \psi(v) \cdot \psi(w)
$$

where $\psi(a)=1$ or $\psi(a)=y$. Hence, by Lemma 3.5 again $\varphi(a) \neq 1$ and $y$ $=a$. Thus:

C) there is a mapping

$$
\psi: X \cup R \cup\{a\} \rightarrow Y \cup S \cup\{a\}
$$

such that $\varphi(x) \geqq \psi(x)$ for each $x \in X \cup R \cup\{a\}, \psi(X) \subset Y, \psi(R) \subset S$, $\psi(a)=a, \psi / X$ is a compatible mapping from $(X, R)$ to $(Y, S)$ such that

$$
\psi(\{u, v\})=\{\psi(u), \psi(v)\} \quad \text { for each }\{u, v\} \in R .
$$

If $\varphi(x)=\psi(x)$ for each $x \in X \cup R \cup\{a\}$ then $\varphi=\Phi(\psi / X)$.

To prove that $\varphi(x)=\psi(x)$ for each $x \in X \cup R \cup\{a\}$ we restrict $\Phi$ to the full subcategory $\mathbf{C}$ of Gra given by Theorem 2.6. By b2) each graph in $\mathbf{C}$ has no isolated point and by b3) each edge is in a triangle. Assume that 
$\varphi(x)>\psi(x)$ for some $x \in X$. Then by b2) there is an infinite subset $T_{x}$ of $X$ with $\{x, z\} \in R$ for each $z \in T_{x}$ such that $\psi / T_{x}$ is one-to-one and for each $y \in Y-\{\psi(x)\}$, and for each pair $u, v \in T_{x}, u \neq v$ we have either

$$
\{\psi(u), y\} \notin S \text { or }\{\psi(v), y\} \notin S .
$$

Thus Lemma 3.6 applies to $\psi\left(T_{x}\right)$ for $(Y, S)$ and $\psi(x)$. Therefore there is $z$ $\in \psi\left(T_{x}\right)$ with

$$
\varphi(x) \cdot z \cdot e \neq 0 \text { for } e=\{z, \psi(x)\} \in S .
$$

There is $t \in T_{x}$ with $\psi(t)=z$, then for $e^{\prime}=\{t, x\} \in R$ we have $\psi\left(e^{\prime}\right)=e$ and hence

$$
0=\varphi\left(x \cdot t \cdot e^{\prime}\right)=\varphi(x) \cdot \varphi(t) \cdot \varphi\left(e^{\prime}\right) \geqq \varphi(x) \cdot z \cdot e \neq 0,
$$

a contradiction. Thus $\varphi(x)=\psi(x)$ for each $x \in X$. Assume that $\varphi(e)>$ $\psi(e)$ for some $e \in R$. Then by b3) there is an infinite subset $T$ of $X$ such that $\{u, z\},\{v, z\} \in R$ for each $z \in T$ where $e=\{u, v\}$ and $\psi$ is one-to-one on $T$. By Lemma 3.7 there is $w \in \psi(T)$ such that for $e_{1}=$ $\{\psi(u), w\}, e_{2}=\{\psi(v), w\} \in S$ we have

$$
\varphi(e) \cdot e_{1} \cdot e_{2} \neq 0 \text {. }
$$

For $z \in T$ with $\psi(z)=w$ and for $e_{1}^{\prime}=\{u, z\}, e_{2}^{\prime}=\{v, z\} \in R$ we have $\psi\left(e_{1}^{\prime}\right)=e_{1}, \psi\left(e_{2}^{\prime}\right)=e_{2}$. Then

$$
\begin{aligned}
0 & =\varphi\left(e \cdot e_{1}^{\prime} \cdot e_{2}^{\prime}\right)=\varphi(e) \cdot \varphi\left(e_{1}^{\prime}\right) \cdot \varphi\left(e_{2}^{\prime}\right) \geqq \varphi(e) \cdot \psi\left(e_{1}^{\prime}\right) \cdot \psi\left(e_{2}^{\prime}\right) \\
& =\varphi(e) \cdot e_{1} \cdot e_{2} \neq 0,
\end{aligned}
$$

a contradiction. Thus $\varphi(e)=\psi(e)$ for each $e \in R$. Assume that $\varphi(a)>a$. Then by b1) there is an infinite set $T$ of triangles in $(X, R)$ such that for each pair of distinct triangles $\{x, y, z\}$ and $\{u, v, w\}$ in $T$,

$$
\{\psi(x), \psi(y), \psi(z)\} \cap\{\psi(u), \psi(v), \psi(w)\}=\emptyset
$$

thus we can use Lemma 3.8 on $\psi(T)$ and hence there is a triangle $\{x, y, z\}$ in $T$ with

$$
\varphi(a) \cdot \psi(x) \cdot \psi(y) \cdot \psi(z) \neq 0
$$

this is a contradiction. Thus $\varphi(a)=\psi(a)$. The proof is complete.

4. Lattice of subvarieties of $\mathscr{A}(B)_{*}$ and $\mathscr{A}(B)^{*}$. We describe the lattice of subvarieties of $\mathscr{A}(B)_{*}$; the description of the lattice of subvarieties of $\mathscr{A}(B)^{*}$ is analogous and we left it for the reader. By [1] we have

Proposition 4.1. $\mathscr{A}(B)_{*}$ is locally finite.

Hence each subvariety of $\mathscr{A}(B) *$ is uniquely determined by its finite subdirectly irreducible lattices. We use Proposition 3.1 to describe 
finite subdirectly irreducible lattices in $\mathscr{A}(B)_{*}$. Observing that every proper principal ideal in $I_{*}(\mathscr{G})$ is finite for each graph $\mathscr{G}$, by a routine calculation we obtain the following proposition:

Proposition 4.2. Let $\mathscr{G}=(V, E)$ be a complete bipartite graph (with a decomposition $V_{1}, V_{2}$ of $V$ with $E=\left\{\left\{v_{1}, v_{2}\right\} ; v_{1} \in V_{1}, v_{2} \in V_{2}\right\}$ ). Let $L$ be a sublattice of $I_{*}(\mathscr{G})$. Put

$$
\begin{aligned}
I L=\{A \in L-\{1\} ; \exists v \in V(v & \in A \text { and } \\
& \forall B \in L(v \in B \Rightarrow A \subseteq B))\} .
\end{aligned}
$$

Then $I L$ is the set of all join-irreducible elements of $L$ different from $1 . L$ is non-distributive if and only if $|I L|>2$ and there are $A, B \in I L$ with $A \cap V_{1}$ $\neq \emptyset, B \cap V_{2} \neq 0$. If $L$ is non-distributive then IL generates $L$ by joins (thus 1 is not a join-irreducible element $)$.

Proposition 4.3. Let $\mathscr{G}=(V, E)$ be a complete bipartite graph. Let $L$ be a sublattice of $I_{*}(\mathscr{G})$. Then $L$ is subdirectly irreducible if and only if $I L=\emptyset$ or $L$ is non-distributive and IL contains at most one element which is not an atom of $L$.

Proof. Let $V_{1}, V_{2}$ be a decomposition of $V$ with

$$
E=\left\{\left\{v_{1}, v_{2}\right\} ; v_{1} \in V_{1}, v_{2} \in V_{2}\right\} \text {. }
$$

Put $I L_{1}=\left\{A \in I L ; A \subset V_{1}\right\}, I L_{2}=\left\{A \in I L ; A \subset V_{2}\right\}$, then if $L$ is non-distributive then $I L_{1} \neq \emptyset \neq I L_{2}$ and $I L=I L_{1} \cup I L_{2}$. Moreover, if $\sim$ is a congruence on $L$ then if $A \sim 0$ for some $A \in I L_{1}$, then $B \sim 1$ for each $B \in I L_{2}$ and $B \sim 0$ for each $B \in I L_{1}$. On the other hand, for each non-identical congruence $\sim$ on $L$ there is $A \in I L$ and $B \subseteq A, B \neq A$ with $B \sim A$ (indeed, if $C \sim D$ for some $C<D \in L$ then there is $A \in I L$ with $A<D$ but $A \nless C$ else $I L$ does not generate $L$ by joins, thus $A \sim A \cap C$ $<A$ ); thus if $L$ is non-distributive and $I L$ contains at most one element which is not an atom of $L$, then $L$ is subdirectly irreducible. Since each proper principal ideal is finite, if $A \in I L$ is not an atom in $L$ then there is the biggest element $B<A$. It is easy to verify that $\sim_{A}$ is a congruence where $C \sim \sim_{A} D$ if and only if $C=D$ or $B \subseteq C$ and $D=C \cup A$ or $B \subseteq D$ and $C=D \cup A$. If $A^{\prime}$ is another non-atomic element of $I L$ then $\sim_{A}$ and $\sim_{A^{\prime}}$ are disjoint congruences and $L$ is not subdirectly irreducible. The rest follows from Proposition 4.2 and from the fact that the two-element lattice is the only distributive subdirectly irreducible lattice.

Let $\mathscr{G}$ be a complete bipartite graph, $L$ a non-distributive subdirectly irreducible sublattice of $I_{*}(\mathscr{G})$; denote by $a I L_{1}$ the set of all atoms in $I L_{1}$ which are maximal elements in $I L_{1}$, and by $a I L_{2}$ the set of all atoms in $I L_{2}$ which are maximal elements in $I L_{2}$ (then by Proposition 4.3 either $I L_{1}=$ $a I L_{1}$ or $\left.I L_{2}=a I L_{2}\right)$. It is an easy exercise to verify: 
Proposition 4.4. Let $\mathscr{G}, \mathscr{H}$ be finite complete bipartite graphs, $L$ (or $M)$ a non-distributive subdirectly irreducible sublattice of $I_{*}(\mathscr{G})\left(\right.$ or $\left.I_{*}(\mathscr{H})\right)$. Then $L$ is isomorphic to $M$ if and only if either $\left|I L_{1}\right|=\left|I M_{1}\right|,\left|a I L_{1}\right|=\left|a I M_{1}\right|$, $\left|I L_{2}\right|=\left|I M_{2}\right|$ and $\left|a I L_{2}\right|=\left|a I M_{2}\right|$, or $\left|I L_{1}\right|=\left|I M_{2}\right|,\left|a I L_{1}\right|=\left|a I M_{2}\right|,\left|I L_{2}\right|$ $=\left|I M_{1}\right|$ and $\left|a I L_{2}\right|=\left|a I M_{1}\right|$.

Let $L$ be a finite subdirectly irreducible non-distributive lattice in $\mathscr{A}(B)_{*}$. Combining Propositions 3.1, 4.3 and 4.4 we get that $L$ is uniquely determined by numbers $\left|I L_{1}\right|,\left|a I L_{1}\right|,\left|I L_{2}\right|,\left|a I L_{2}\right|$. We can assume that either $\left|I L_{1}\right|>\left|I L_{2}\right|$ or $\left|I L_{1}\right|=\left|I L_{2}\right|$ and $\left|a I L_{1}\right| \geqq\left|a I L_{2}\right|$. Denote $L$ as $K\left(\left|I L_{1}\right|,\left|a I L_{1}\right|,\left|I L_{2}\right|,\left|a I L_{2}\right|\right)$. Then we have:

PROPOSITION 4.5. a) $K(m, n, p, q)$ is a sublattice of $K\left(m^{\prime}, n^{\prime}, p^{\prime}, q^{\prime}\right)$ if and only if $m<m^{\prime}$ or $m=m^{\prime}$ and $n \leqq n^{\prime}$ and at the same time $p<p^{\prime}$ or $p=p^{\prime}$ and $q \leqq q^{\prime}$.

b) $K(m, n, p, q)$ is simple if and only if $m=n$ and $p=q>1$.

c) If $K(m, n, p, q)$ is not simple and if $\sim$ is the smallest congruence on $K(m, n, p, q)$ then

$$
\begin{aligned}
& K(m, n, p, q) / \sim=K(m-1, m-1, p, q) \quad \text { if } m>n \\
& K(m, n, p, q) / \sim=K(m, n, p-1, p-1) \quad \text { if } p>q,
\end{aligned}
$$

$K(m, n, p, q) / \sim$ is the two-element lattice if $m=n, p=q=1$.

Proof. Proof of a) is a routine calculation, b) follows from [1] and the proof of Proposition 4.3, c) follows from the proof of Proposition 4.3.

Put $C=\{(m, n, p, q) ; m, n, p, q$ are positive integers with $m \geqq n, m \geqq p$ $\geqq q$ and either $m=n$, or $p=q$, and $m \neq n$ implies $m>p\}$ and define an ordering $\leqq$ on $C$ such that $(m, n, p, q) \geqq\left(m^{\prime}, n^{\prime}, p^{\prime}, q^{\prime}\right)$ if either $m>m^{\prime}$ or $m=m^{\prime}$ and $n \geqq n^{\prime}$ and at the same time either $p>p^{\prime}$ or $p=p^{\prime}$ and $q \geqq$ $q^{\prime}$. If we identify $K(1,1,1,1)$ with the two-element lattice then we get:

Corollary 4.6. a) If $\alpha, \beta \in C$ then the variety of lattices generated by $K(\alpha)$ contains $K(\beta)$ if and only if $\alpha \geqq \beta$.

b) There is a bijective correspondence $\psi$ between subvarieties of $\mathscr{A}(B) *$ and order-ideals of $C$; for a subvariety $\mathscr{V}$ of $\mathscr{A}(B)_{*}, \psi(\mathscr{V})$ is the set of all $\alpha \in C$ with $K(\alpha) \in \mathscr{V}$.

The proof immediately follows from Proposition 4.5 and the Jonsson Lemma see $[8]$ or $[4]$.

We shall extend $C$ on

$$
\bar{C}=C \cup\{(\infty, p, q) ; p \geqq q \text { are positive integers }\} \cup\{(\infty, \infty)\}
$$

and we extend the ordering $\leqq$ such that $(\infty, p, q) \geqq\left(m^{\prime}, n^{\prime}, p^{\prime}, q^{\prime}\right)$ or $\geqq$ $\left(\infty p^{\prime}, q^{\prime}\right)$ if $p>p^{\prime}$ or $p=p^{\prime}$ and $q \geqq q^{\prime}$ and $(\infty, \infty)$ is the largest element of $C$. 
LEMMA 4.7. a) There is a bijective correspondence $\Phi$ between independent subsets of $\bar{C}$ and order-ideals of $C$ such that for an independent subset $A$ of $\bar{C}$,

$$
\Phi(A)=\{\alpha \in C ; \exists \beta \in A, \alpha \leqq \beta\} .
$$

b) Each independent set in $\bar{C}$ is finite.

The proof is a routine calculation.

Let $p$ be a positive integer, let $(V, E)$ be a complete bipartite graph with a decomposition $V_{1}, V_{2}$ of $V$ such that

$$
\begin{aligned}
& E=\left\{\left\{v_{1}, v_{2}\right\} ; v_{1} \in V_{1}, v_{2} \in V_{2}\right\}, \\
& \left|V_{1}\right|=\aleph_{0}, \\
& V_{2}=\left\{w_{0}, w_{1}, \ldots, w_{p-1}\right\} .
\end{aligned}
$$

For $q<p$ define a sublattice of $L_{q}$ of $I_{*}(V, E)$ generated by the set consisting from all singletons in $V$ except $\left\{w_{p-1}\right\}$ and from the set $\left\{w_{q}\right.$, $\left.w_{q+1}, \ldots, w_{p-1}\right\}$. Then by Proposition $4.3 L_{q}$ is subdirectly irreducible. Denote $L_{q}=K(\infty, p, q)$, further denote $I_{*}(V, E)=K(\infty, p, p)$ and $I_{*}(W, F)=K(\infty, \infty)$ where $(W, F)$ is a complete bipartite graph with a decomposition $W_{1}, W_{2}$ of $W$ with

$$
\begin{aligned}
& F=\left\{\left\{v_{1}, v_{2}\right\} ; v_{1} \in W_{1}, v_{2} \in W_{2}\right\} \text { and } \\
& \left|W_{1}\right|=\left|W_{2}\right|=\aleph_{0} .
\end{aligned}
$$

Then we get:

THEOREM 4.8. a) For each subvariety $\mathscr{V}$ of $\mathscr{A}(B)_{*}$ there is (exactly one) a (finite) independent set $A_{\mathscr{V}}$ in $\bar{C}$ such that $\left\{K(\alpha) ; \alpha \in A_{\mathscr{V}}\right\}$ generates $\mathscr{V}$ (in the sense of Birkhoff theorem). Moreover, for a pair of subvarieties $\mathscr{V}_{1}, \mathscr{V}_{2}$ of $\mathscr{A}(B)_{*}, \mathscr{V}_{1} \subset \mathscr{V}_{2}$ if and only if for each $\alpha \in A_{\mathscr{V}_{1}}$ there is $\beta \in A_{\mathscr{V}_{2}}$ with $\alpha \leqq$ $\beta$.

b) The lattice of subvarieties of $\mathscr{A}(B)_{*}$ is countable.

An analogous theorem holds in $\mathscr{A}(B)^{*}$; we have only to substitute $I^{*}(\mathscr{G})$ for $I_{*}(\mathscr{G})$ (for any complete bipartite graph $\mathscr{G}$ ). Hence we get:

Corollary 4.9. $\mathscr{A}(B)_{*} \cap \mathscr{A}(B) *$ is the variety generated by $N_{5}=K(2$, $0,1,1)$.

Since each non-trivial lattice in the variety $\mathscr{V}$ generated by $N_{5}$ has a prime ideal, no non-trivial group is an endomorphism monoid of a lattice in $\mathscr{V}$. Thus:

COROLlary 4.10. The smallest binding variety of lattices does not exist. 
Furthermore, there is no smallest variety $\mathscr{V}$ for which every monoid is isomorphic to an endomorphism monoid of a lattice in $\mathscr{V}$.

I wish to acknowledge helpful discussions with P. Goralč́k and J. Sichler.

\section{REFERENCES}

1. M. E. Adams and J. Sichler, Cover set lattices, Can. J. Math. 32 (1980), 1177-1205.

2. M. E. Adams, A. Day and J. Sichler, Small lattice varieties generated by cover set lattices, to appear.

3. M. E. Adams, D. Pigozzi and J. Sichler, Endomorphisms of direct unions of bounded lattices, Archiv. Math. 36 (1981), 221-229.

4. G. Grätzer, General lattice theory (Academic Press, New York, San Francisco, 1978).

5. G. Grätzer and J. Sichler, On the endomorphism semigroup (and category) of bounded lattices, Pacif. J. Math. 35 (1970), 639-647.

6. Z. Hedrlín and A. Pultr, On full embeddings of categories of algebras, Illinois J. Math. 10 (1966), 392-406.

7. Z. Hedrlín and J. Sichler, Any boundable binding category contains a proper class of mutually disjoint copies of itself, Algebra Universalis 1 (1971), 97-103.

8. B. Jónsson, Algebras whose congruence lattices are distributive, Math. Scan. 2 ( 1967), $110-121$.

9. V. Koubek, Graphs with given subgraphs represent all categories, Comment. Math. Univ. Carolinae 18 (1977), 115-127.

10. Graphs with given subgraphs represent all categories II, Comment. Math. Univ. Carolinae 19 (1978), 249-264.

11. V. Koubek and J. Sichler, Preservation properties of full embeddings, to appear.

12. E. Mendelsohn, On technique for representing semigroups and endomorphism semigroups of graphs with given properties, Semigroup Forum 4 (1972), 283-294.

13. A. Pultr and V. Trnková, Combinatorial, algebraic and topological representations of groups, semigroups and categories (North Holland, Amsterdam, New York, Oxford, 1980).

Computational Centre of Charles University,

Malostranské nám. 25, Praha 1, Czechoslovakia 